\title{
A magnetic compatible supernumerary robotic finger for functional magnetic resonance imaging (fMRI) acquisitions: device description and preliminary results.
}

\author{
Irfan Hussain ${ }^{1}$, Emiliano Santarnecchi ${ }^{2,4}$, Andrea Leo $^{5}$, Emiliano Ricciardi ${ }^{6}$, Simone Rossi ${ }^{2,3}$ \\ and Domenico Prattichizzo ${ }^{1,7}$
}

\begin{abstract}
The Supernumerary robotic limbs are a recently introduced class of wearable robots that, differently from traditional prostheses and exoskeletons, aim at adding extra effectors (i.e., arms, legs, or fingers) to the human user, rather than substituting or enhancing the natural ones. However, it is still undefined whether the use of supernumerary robotic limbs could specifically lead to neural modifications in brain dynamics. The illusion of owning the part of body has been already proven in many experimental observations, such as those relying on multisensory integration (e.g., rubber hand illusion), prosthesis and even on virtual reality. In this paper we present a description of a novel magnetic compatible supernumerary robotic finger together with preliminary observations from two functional magnetic resonance imaging (fMRI) experiments, in which brain activity was measured before and after a period of training with the robotic device, and during the use of the novel MRI-compatible version of the supernumerary robotic finger. Results showed that the usage of the MRcompatible robotic finger is safe and does not produce artifacts on MRI images. Moreover, the training with the supernumerary robotic finger recruits a network of motor-related cortical regions (i.e. primary and supplementary motor areas), hence the same motor network of a fully physiological voluntary motor gestures.
\end{abstract}

\section{INTRODUCTION}

Long-term disabilities of the upper limb affects millions of stroke survivors [1]. More than $80 \%$ of individuals who experience severe hemiparesis after stroke cannot completely recover hand and arm use [2]. Those who are left with single functional hand face problems in performing even simple activity daily living (ADL) tasks, specially bi-manual. Existing compensatory robotic devices like prostheses, cannot be used to help them since the hand of the patient, although frequently with limited mobility, is still present. Early results on the replacement of impaired hand with robotic devices are reported in [3]. However, this potential solution could

\footnotetext{
${ }^{1}$ Dept. of Information Engineering and Mathematics, University of Siena, Via Roma 56, 53100 Siena, Italy. $\{$ irfan.hussain\}@unisi.it

2 Department of Medicine, Surgery and Neuroscience, Brain Investigation and Neuromodulation Lab, Unit of neurology and Clinical Neurophysiology, University of Siena, Siena, Italy.

${ }^{3}$ Deparment of Medicine, Surgery and Neuroscience, Human Physiology Section, University of Siena, University of Siena, Siena, Italy.

${ }^{4}$ Berenson-Allen Center for Non-invasive Brain Stimulation, Division of Cognitive Neurology, Department of Neurology, Harvard Medical School, Boston, MA, USA.

${ }^{5}$ Research Center E. Piaggio, University of Pisa, Pisa, Italy.

${ }^{6}$ MOMILab, IMT School for Advanced Studies Lucca, Lucca, 55100, Italy.

${ }^{7}$ Department of Advanced Robotics, Istituto Italiano di Tecnologia, Via Morego 30, Genoa, Italy.
}

be much less effective in chronic stroke patients where the whole arm often present a limited residual mobility. On contrary, rigid exoskeletons do not accommodate variations in patient skeletal structure or joint misalignment and can produce compression forces on the soft tissue and joints during long-term use [4]. Moreover, most of the proposed exoskeletons are quite cumbersome, thus limiting the wearability and portability of the device. Besides exoskeletons and prostheses and their working principles, other robotic solutions which can compensate the missing grasping function are needed. Towards this aim, in [5], [6], [7], authors presented the supernumerary robotic fingers to augment the capabilities of healthy hand in order to enable it to do the tasks which normally require two hands (e.g. grasping bigger size objects, opening a cap of bottle etc). As an alternative approach, in [8], [9], [10] we proposed the use of a supernumerary robotic finger as an active compensatory tool for chronic stroke patients. The ultimate objective of the research is to achieve as much as possible the natural interaction of humans with supernumerary robotic devices, so that they can control these devices as if they actually belong to them. One interesting question that needs to be addressed is whether the practice with supernumerary robotic leads to plastic modifications in brain activity; additional questions may regard the embodiment or the perception of extra limbs as if they belong to the subjects body. Such effect has been proved in the past both with realistic limbs (i.e., rubber hands) and six-fingers avatars controlled using virtual reality [11], [12]. In 2011, Guterstam et al. [13] demonstrated the possibility of inducing the perceptual illusion of having a supernumerary right hand. Their findings suggest that the neural embodiment of a supernumerary limb is possible if it is aligned with the body in an anatomically similar fashion as the real limbs. In addition, it has been demonstrated that the size of the incorporated body part is not important and ownership illusion can be induced towards very small or large bodies [14]. Other studies showed that although multiple supernumerary limbs can be incorporated into the bodily image (i.e. the sense of ownership towards the supernumerary limb), only one can be included in the body schema (i.e. the ability to control the supernumerary limb) [15], [16]. This is why the study of brain activity during the use of supernumerary robotic limbs is a topic of particular interest. We designed and developed both MRI compatible and non-compatible robotic fingers to be used both inside and outside the fMRI scanner during everyday 
activities: so, either task related and training or use related brain activity can be investigated. To develop more complex experimental fMRI paradigm, it is a great interest to realize active interfaces, using electrically powered actuators and sensors to be used inside the MRI environment [17]. The use of non-ferromagnetic metals with higher stiffness and rigidity compared to plastic facilitates the design of smaller devices. In literature, several reports provide criteria to both develop and test MR compatible devices [18], [19]. Indeed, specific experimental protocols are described to evaluate compatibility and to evaluate location and timing zones where MR compatibility should be assessed. The various phenomena that can happen when a mechatronic device is placed adjacent to MR scanner should be taken into account. MR compatibility needs a set of supplementary constraints in the actuator and sensor choice and requires a proper design process. The common standard mechanical parts cannot be used in the MR environment because they usually contain ferromagnetic components. Majority of the actuators are electromagnetic devices and practically impossible to use during imaging. We took all these considerations into account during the design and development of our MR compatible supernumerary robotic finger.

The rest of the paper is organized as it follows. Section II describes the design and development of MR compatible supernumerary robotic finger while in Section III the MR non-compatible finger is detailed. Section IV deals with the preliminary fMRI experiments that assessed the changes in neural responses associated to the usage of the supernumerary finger inside the scanner or to three days training with the supernumerary robotic finger outside the scanner. The results and discussions are outlined in Section V. Finally, Section VI presents the conclusion and future work.

\section{MRI COMPATIBLE SUPERNUMERARY ROBOTIC FINGER}

We developed an MRI compatible finger to be used inside an fMRI scanner. Functional brain exploration methodologies, such as fMRI are at present used to study brain correlates of perceptual, motor and cognitive processes.

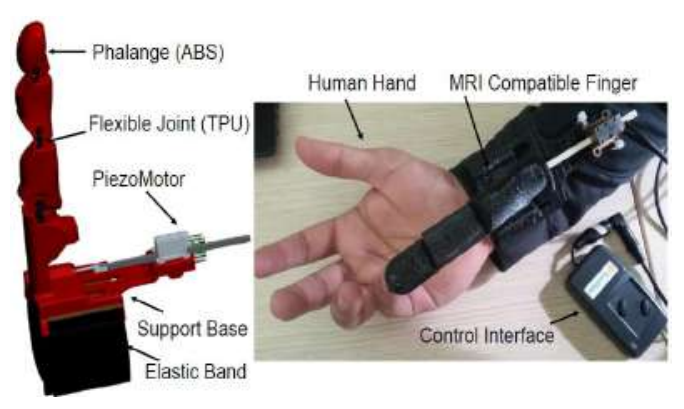

Fig. 1. 3D printed MRI compatible finger having flexible material (TPU) as flexible joints and stiff (ABS) material acting as rigid links. The actuator used is a piezoelectric motor.

We developed the MRI compatible robotic finger for fMRI experiments by properly choosing materials and actuators whose properties are fully compliant to MR compatibility.
The proposed device consists of two main parts, a flexible finger and a support base, see figure 1. Each phalange of the finger has a 3D printed ABS (Acrylonitrile Butadiene Styrene, ABSPlus, Stratasys, USA) polymeric part that acts as a rigid link and a 3D printed thermoplastic polyurethane (Lulzbot, USA) part that realizes the flexible joint. The phalanges are connected by sliding the thermoplastic polyurethane part in the ABS part. A single actuator used to move the whole finger through a tendon. A hole in the rigid link allows the passage of the cable (polyethylene dyneema fiber, Japan) which is used to realize the tendon driven actuation. The tendon wire runs through the finger and is attached on one side to the fingertip and on the other to the shaft of the actuator. Due to flexible parts in the joints, the device is passively compliant. Reasons for adding passive elements are manifold, including avoiding tendon slackness and ensuring the uniqueness of the position [20]. The builtin compliant nature of the extra-finger increases its ability to grasp different objects. The support base of the finger is realized in ABS. It contains the actuator and an elastic band that allows the user to wear the device on the forearm. The structure of the support base is symmetrical, a feature that enables the robotic finger to be worn on both the left or right hand of the user without any modification in the device.

The actuator used is a piezo LEGS linear motor (linear twin $20 N$, PiezoMotor, Swedon) which can provide high force output in a very compact body. Principal details on the motor features are reported in Table I. It features predictable sub-micron, direct drive motion without backlash with resolution down to single nanometer level or below. Lack of gear heads and linear screws reduce the overall size of the motor. It can deliver up to $20 \mathrm{~N}$ force. The drive technology is direct, meaning no gears or lead screws are needed to create linear motion. The motor has no mechanical play or backlash.

The Piezo LEGS walking principle is of the non-resonant type, i.e. the position of the drive legs is known at any given moment. This assures very good control of the motion over the whole speed range. The performance of a Piezo LEGS motor is different from that of a DC or stepper motor in several aspects. A Piezo LEGS motor is friction based, meaning the motion is transferred through contact friction between the drive leg and the drive rod.

The controller used is hand-held driver PMCM21 (PiezoMotor, Swedon) which is a simple hand-held pushbutton driver for Piezo LEGS motors that offers sub-micron resolution linear motion. Pushing the button to move in one direction ramps up speed by utilizing phase-shifting of the drive signal. A quick press of the button generates submicron steps. Positioning below $0.1 \mathrm{~m}$ is possible by altering direction.

\section{MRI NON MAGNETIC COMPATIBLE SUPERNUMERARY ROBOTIC FINGER}

The non magnetic compatible supernumerary robotic finger as shown in Fig. 2 is composed by four one-DoF modules. Each module consists of a servomotor (HS-53 Microservo, HiTech, Republic of Korea), a 3D printed plastic 
TABLE I

THE MRI COMPATIBLE SUPERNUMERARY ROBOTIC FINGER TECHNICAL DETAILS

\begin{tabular}{|l|r|}
\hline Phalange dimensions & $2.0 \times 3.1 \times 1.2 \mathrm{~cm}^{3}$ \\
\hline Support base dimensions & $6.4 \times 7.0 \times 0.35 \mathrm{~cm}^{3}$ \\
\hline Total weight: finger + support base & $140 \mathrm{~g}$ \\
\hline \hline Actuator maximum stroke & $00 \mathrm{~mm}$ \\
\hline Actuator speed range & $20 \mathrm{~mm} / \mathrm{s}$ \\
\hline Actuator stall force & $22 \times 21 \times 10.8 \mathrm{~mm}^{3}$ \\
\hline Actuator dimensions & $29 \mathrm{~g}$ \\
\hline Actuator weight & Hand-held driver $($ PMCM21) \\
\hline \hline Control & \\
\hline
\end{tabular}

TABLE II

MRI NON-COMPATIBLE SUPERNUMERARY ROBOTIC FINGER TECHNICAL DETAILS

\begin{tabular}{|l|r|}
\hline Module dimension & $42 \times 33 \times 20 \mathrm{~mm}^{3}$ \\
\hline Support base dimension & $78 \times 24 \times 5 \mathrm{~mm}^{3}$ \\
\hline \hline Module weight & $8 \mathrm{~g}$ \\
\hline Support base weight & $18 \mathrm{~g}$ \\
\hline \hline Max torque per motor & $0.15 \mathrm{Nm}$ \\
\hline Stall current & $440 \mathrm{~mA}$ \\
\hline Velocity of one module & $0.2 \mathrm{rad} / \mathrm{s}$ \\
\hline \hline Device external batteries & $7.5 \mathrm{~V}, 2.2 \mathrm{Ah}$ \\
\hline \hline Continuous operating time & $3.5 \mathrm{~h}(@$ stall torque $)$ \\
\hline Max device payload & $610 \mathrm{~g}$ \\
\hline
\end{tabular}

part (acrylonitrile butadiene styrene, ABSPlus, Stratasys, USA) and a soft rubber part used to increase the friction at the contact area. The modules are connected so that one extremity of each module is rigidly coupled with the shaft of the motor through screws, while the other has a pin joint acting as revolute joint. The module connection results in a pitch-pitch configuration, which replicates the flexion/extension motion of the human finger. The detail design of the device is presented in [21] while the technical features are reported in Table II.

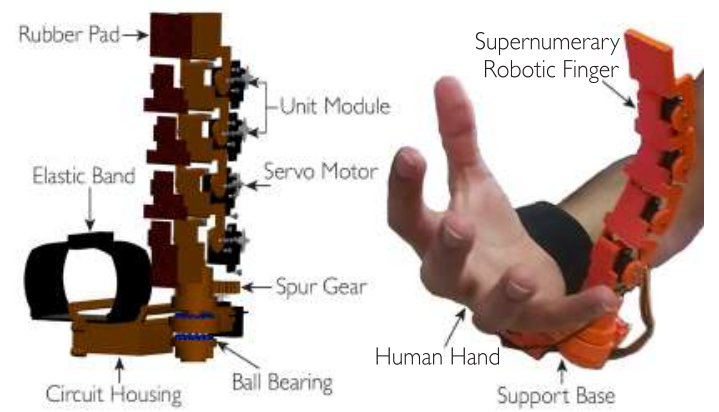

Fig. 2. The non MRI compatible supernumerary robotic finger. The device exploded CAD view and prototype are shown. It can be worn on the forearm thanks to an elastic band. When activated, it interacts with the hand in grasping tasks.

The modular part of the finger is connected to a support base which contains also the electronic housing. An elastic band allows to easily wear the device on the forearm. An external battery is used to provide power to all the circuits. All the electronics is enclosed in a 3D printed box attached to the support base to preserve the extra-finger wearability.
The module actuators are PWM controlled servomotors. The PWM signals are generated by an Arduino Nano board [22]. One of the major challenge in augmenting the human hand capabilities through supernumerary robotic fingers lies in developing a suitable control interface and in designing algorithms for seamless integration of device motion with that of human hand. In [7], we presented a mapping algorithm able to transfer to an arbitrary number of the robotic extrafingers the motion of the human hand. We used the same algorithm to control the motion supernumerary robotic finger in coordination with human hand. The Cyberglove III System [23] is used to track the human hand. The dataglove and robotic finger controller are connected through serial ports with a computer running the mapping algorithm.

\section{EXPERIMENTS}

\section{A. Training}

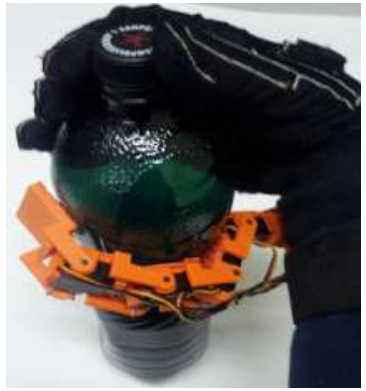

(a) Opening the bottle.

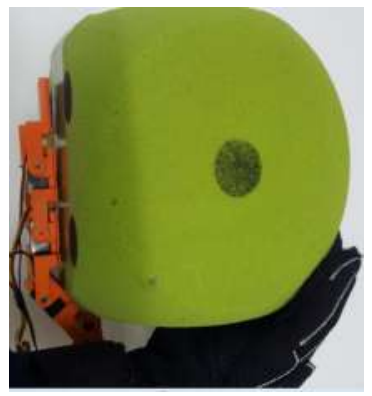

(c) Grasping dice

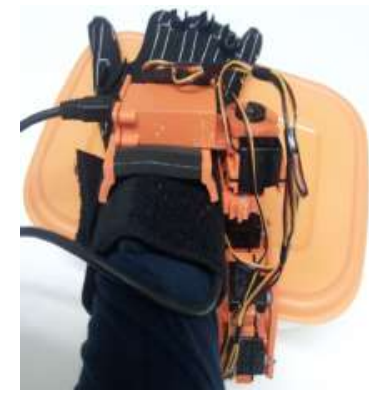

(b) Grasping bigger size box

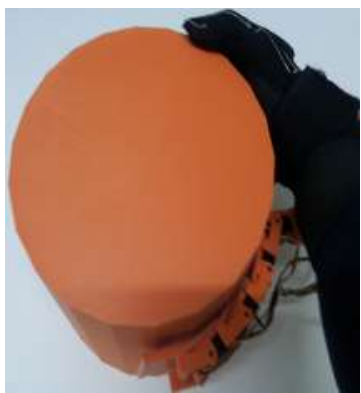

(d) Grasping bigger size cylinder
Fig. 3. Examples of tasks performed during the training with the supernumerary robotic finger.

Nine right-handed healthy subjects (males; mean age range 30-35 years) took part into the training that involved the use of supernumerary robotic finger in activity of daily living (ADL) tasks. The training period consisted in 2 days, four hours per day. The training tasks comprised an extensive practice in grasping target objects of different sizes and shapes (e.g., box, dice, cylinder) with the augmented hand to evaluate the effectiveness of the extra robotic finger in enlarging the human hand workspace and its dexterity. Participants also performed manipulation tasks that usually involve both hands. Fig. 3 shows examples of motor tasks performed with the help of the supernumerary robotic finger using the sensorized glove. Note that in Fig. 3-a, the supernumerary finger is used in coordination with the little and ring finger, 
while in the other two examples, the robotic finger is used in coordination with the whole hand to hold large size objects. To this aim, the agent fully opens his hand and the extra finger comes in the middle of the human hand with all the joints fully extended. After that, once the human hand moves to grasp the object, the extra-finger movement is coordinated with the movement of the real fingers. Of note, all the tasks are either anatomically and physiologically impossible or at least very difficult to be carried out with a single hand. The augmented hand is able to grasp larger size objects, which are impossible to be grasped if the hand is unaided.

\section{B. MRI data acquisition}

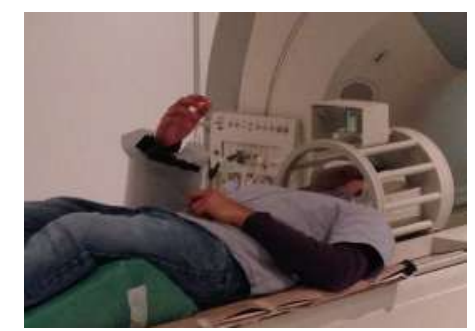

Fig. 4. The experimental fMRI setup:The subject is using MRI compatible finger inside the fMRI scanner.

Participants performed three different experimental sessions: one before the beginning and one at the end of the two days training period. Immediately after the second (posttraining) session, subjects performed a third fMRI acquisition using the MR-compatible device inside the scanner. For the three sessions, a common experimental paradigm, involving a simple finger-tapping task, was adopted: participants had to tap their fingers (from II to V) against the thumb, with a frequency of about $1 \mathrm{~Hz}$, for 20 seconds. The blocks were interleaved with a resting period of 20 seconds. The task blocks were performed in a fixed order (II-III-IV-V) and the total duration of each run was 3 minutes (m) 40 seconds(s), including two 20s-long rest periods at the beginning and at the end. Four runs were performed, with the right hand (odd runs) and left hand (even runs), respectively. Immediately after the second (post-training) session, subjects performed a third acquisition using the MR compatible device for both hands.

\section{Methods}

Data were acquired on a Philips 1.5 Tesla Intera scanner, equipped with a 16 channels head coil. Functional runs (Gradient Echo EPI) were acquired using these parameters: FieldOf-View 218x218 mm, matrix 128x128, voxel size 1.7x1.7x3 $\mathrm{mm}$, axial acquisition, 80 volumes, 23 slices, Repetition Time: 2.5 s, Echo Time: 40 ms; Flip Angle: 30. The volume of interest included the primary motor, somatosensory and parietal areas. Additional whole brain structural images (3D T1-weighted) were acquired (matrix 256x256x150, voxel size $1 \mathrm{~mm}^{3}$, axial acquisition, Repetition Time: $30 \mathrm{~ms}$, Echo Time: 30 ms; Flip Angle: 40. A T2 weighted Turbo Fluid Attenuated Inversion Recovery (FLAIR) 3-mm thick axial image was also acquired to ensure no participants display brain lesions.

Data from the post-training session of one subject and from the session with the MR compatible device of three subjects were discarded for acquisition problems and excessive head motion, respectively. Functional MRI data were analyzed with the AFNI and FSL software packages [24], [25]. Preprocessing included slice timing correction, spike removal, motion correction, estimation of motion outliers (fsl motion outliers), threshold $6 \mathrm{~mm}$ [26], spatial smoothing (FWHM $5 \mathrm{~mm}$ ) and normalization of time series. Later, a multiple linear regression with a model for the temporal autocorrelation of fMRI time series (ARMA 1,1) was used to estimate the BOLD responses for each task block. The hemodynamic responses for each block (i.e., each digit) were modeled individually as regressors of interest, while polynomial trends, motion parameters and spikes were modeled as regressors of no-interest. Structural images were transformed to MNI152 space with a nonlinear registration method (3dQwarp) and the resulting matrix was also applied to register the t-score maps from the functional data. Two separate group analyses were performed, to evaluate the impact of the training and to evaluate the brain regions that are active during the usage of the MR-compatible supernumerary robotic finger. For the pre-and post-training sessions, coefficients were analyzed with a 3-way analysis of variance (ANOVA), with session (pre- and post-training) and digits (II to V) as fixed factors. For the session with the MR compatible device, data were analyzed through a one-sample Mixed Effect Meta-Analysis (MEMA). Cluster correction was applied to all the fMRI results using Monte Carlo simulations (3dClustSim, 10,000 iterations). The minimum cluster size for a corrected p-value of 0.05 was 166 voxels.

\section{RESUlTS AND Discussion}

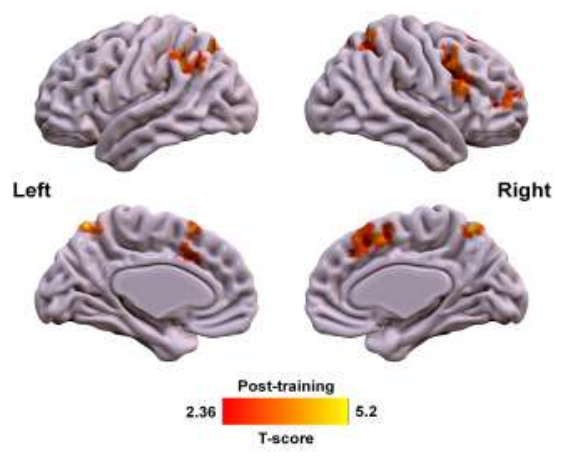

Fig. 5. Pre V/s post trainging contract (ANOVA).

In this study, we presented a novel MR compatible finger together with preliminary fMRI results. MR compatibility needs a set of supplementary constraints in the material and actuator which needs a proper design and development process. The common standard material and mechanical parts cannot be used in magnetic resonance environment because they usually contain ferromagnetic components. Majority of the actuators are electromagnetic devices and practically 


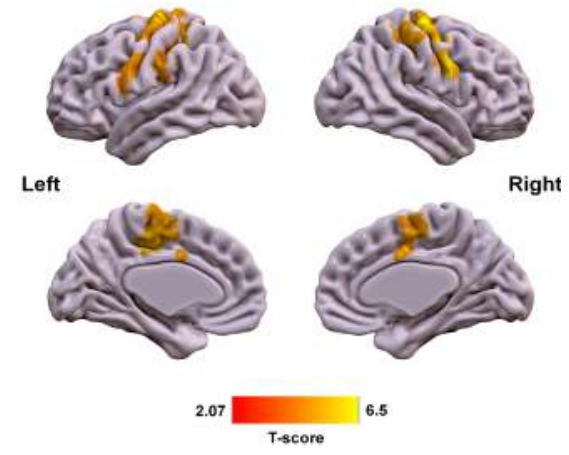

Fig. 6. The brain region activated during the task with the MRI compatible finger

impossible to use during imaging. We realized the MRI compatible finger by using suitable materials and actuator. In particular, the device is $3 \mathrm{D}$ printed by using $\mathrm{ABS}$ and thermoplastic polyurethane (TPU) material and actuator used is a piezoelectric motor with non-magnetic housing. The ABS parts act as rigid phalanges and TPU parts play the role of flexible joints. The flexible joints slides inside the rigid link which makes the assembling process of all the parts easy and without any screws or other metal passive elements in the joints. The actuation system of the finger is based on cable driven and underactuation. We used the device in fMRI experiments outlined in this study. The usage of the MRI compatible supernumerary finger was safe inside the scanner, and, just relying on a qualitative assessment of the functional response maps during a motor task, appeared not induce any artifact in MRI reconstruction of the brain. We also presented the MRI non-compatible finger version which is a modular 3D printed structure where each module is composed of a rigid link and a servo motor. The motion of both devices can be controlled by their control interfaces presented in this paper. Developing a suitable strategy for controlling an extra limb, whether it is a finger or an arm, is of high interest for various fields: for example, in [27] authors studied how a surgeon can use his foot to control an extra arm, at least in a virtual environment. Since it is important that the operator keeps focused on the task he has to perform, the control paradigm of the robot should be felt natural to him, as if the supernumerary limb was part of his body.

In this preliminary fMRI study for supernumerary fingers, greater neural responses for the post-training, as compared to the pre-training condition, were found in a network of brain regions including bilateral cingulate cortex, bilateral superior parietal lobule, left inferior parietal lobule and right middle frontal gyrus (see figure 5). Task-related use of the magnetic compatible supernumerary finger inside the scanner activated a bilateral network of sensorimotor regions, including bilateral primary motor and primary somatosensory areas and supplementary motors areas (SMA) (see Figure 6).

The two day training with extra robotic finger did not induce detectable modifications in the resting state activity of primary sensorimotor areas. This can be due to the small sample size or to the adoption of different tasks for the train- ing (i.e., anatomically impossible grasping gestures) and the fMRI experiment (i.e., a simple finger-to-finger opposition task). Future work should employ a different paradigm for fMRI, with a task involving finalized hand gestures inside the scanner, in order to evaluate the newly acquired motor synergies and their similarity to physiological ones. Activity changes in supplementary motor areas are linked to motor training, even for short periods [28], and it is not surprising to find an enhanced activity of this region. The differences in superior parietal activations (Fig. 5) could be explained with modification in space representation induced by the device, while the results in the left inferior parietal cortex may be driven by the practice with the supernumerary finger, used as a tool [29].

Even if the neuroscientific results are preliminary, mainly due to the small sample size, they suggest that the magnetic compatible supernumerary finger can be safely used inside the scanner, to assess the neural correlates of the rehabilitation with the supernumerary robotic finger use in stroke patients [9], providing the brain functional and structural counterparts of the improvement of everyday life activities which has been observed in previous behavioral experiments. They represent a first, original, step toward a better understanding of brain correlates of the extra finger's use and of the relationship between extra-limbs and their eventual embodiment processes.

\section{CONCLUSIONS AND FUTURE WORK}

The new generation supernumerary robotic limbs is a rapidly growing field and represents a challenging area of research which aims at, adding robotic limbs to human to augment the manipulation abilities of natural limbs. In this direction, an interesting yet challenging issue needs to be addressed, that is: how the human cognitive system perceives, the use of supernumerary robotic limbs. This goal passes through the investigation of brain functional changes due to the use of robotic extra limbs. In this paper, we presented the design and development of our supernumerary robotic fingers having main focus on the new MRI compatible finger. We successfully acquired the fMRI data even in the presence of MRI compatible robotic finger without any artifact in MRI reconstruction of the brain. In future, we are planning to add more degree of freedom in the design of robotic finger to improve its manipulation capabilities. The current study on healthy subjects is a necessary step before the investigation of neural correlates of robotic extra fingers' use in stroke patients, where the supernumerary finger has been already successful for improving everyday patients' life activities [10].

The task related use of the magnetic compatible supernumerary finger inside the scanner activated the same motor network recruited by fully physiolocial voluntary motor gestures (see, Fig. 6), thus implying that the use of the supernumerary robotic finger does not require the acquisition of additional skills or abilities. This justifies the easiness and immediacy of use of the extra robotic finger in stroke patients for grasping [9]. Future studies should 
better disentangle relationships between this brain activity and behavioual outcomes.

Even though, the present results must be considered preliminary, they allow to affirm that the proposed magnetic compatible supernumerary robotic finger is safe even for the use inside the scanner and it does not alter the quality of brain images. This study represents a first, original, step toward a better understanding of brain correlates of the extra robotic finger's use and of the relationship between extra-limbs and their eventual embodiment processes.

\section{ACKNOWLEDGMENT}

The research leading to these results has received funding from the European Union's Horizon 2020 Research and Innovation Programme under Grant Agreement No 688857 of the project SoftPro: Synergy-based Open-source Foundations and Technologies for Prosthetics and RehabilitatiOn.

\section{REFERENCES}

[1] A. S. Go, D. Mozaffarian, V. L. Roger, E. J. Benjamin, J. D. Berry, M. J. Blaha, S. Dai, E. S. Ford, C. S. Fox, S. Franco, et al., "Heart disease and stroke statistics-2014 update: a report from the american heart association.," Circulation, vol. 129, no. 3, p. e28, 2014.

[2] H. Nakayama, H. S. Jorgensen, H. O. Raaschou, and T. S. Olsen, "Compensation in recovery of upper extremity function after stroke: the copenhagen stroke study," Archives of physical medicine and rehabilitation, vol. 75, no. 8, pp. 852-857, 1994.

[3] O. C. Aszmann, A. D. Roche, S. Salminger, T. Paternostro-Sluga, M. Herceg, A. Sturma, C. Hofer, and D. Farina, "Bionic reconstruction to restore hand function after brachial plexus injury: a case series of three patients," The Lancet, vol. 385, no. 9983, pp. 2183-2189, 2015.

[4] J. Pons, E. Rocon, R. Ceres, D. Reynaerts, B. Saro, S. Levin, and W. Van Moorleghem, "The manus-hand dextrous robotics upper limb prosthesis: mechanical and manipulation aspects," Autonomous Robots, vol. 16, no. 2, pp. 143-163, 2004.

[5] F. Wu and H. Asada, "Bio-artificial synergies for grasp posture control of supernumerary robotic fingers," in Proceedings of Robotics: Science and Systems, (Berkeley, USA), July 2014.

[6] D. Prattichizzo, M. Malvezzi, I. Hussain, and G. Salvietti, "The sixthfinger: a modular extra-finger to enhance human hand capabilities," in Proc. IEEE Int. Symp. in Robot and Human Interactive Communication, (Edinburgh, United Kingdom), 2014.

[7] D. Prattichizzo, G. Salvietti, F. Chinello, and M. Malvezzi, "An objectbased mapping algorithm to control wearable robotic extra-fingers," in Proc. IEEE/ASME Int. Conf. on Advanced Intelligent Mechatronics, (Besançon, France), 2014.

[8] I. Hussain, G. Salvietti, L. Meli, C. Pacchierotti, and D. Prattichizzo, "Using the robotic sixth finger and vibrotactile feedback for grasp compensation in chronic stroke patients," in Proc. IEEE/RAS-EMBS International Conference on Rehabilitation Robotics (ICORR), 2015.

[9] G. Salvietti, I. Hussain, D. Cioncoloni, S. Taddei, S. Rossi, and D. Prattichizzo, "Compensating hand function in chronic stroke patients through the robotic sixth finger," Transaction on Neural System and Rehabilitation Engineering, 2016.

[10] I. Hussain, G. Salvietti, G. Spagnoletti, and D. Prattichizzo, "The soft-sixthfinger: a wearable emg controlled robotic extra-finger for grasp compensation in chronic stroke patients," IEEE Robotics and Automation Letters, vol. 1, pp. 1000 -1006, July 2016.

[11] L. Hoyet, F. Argelaguet, C. Nicole, and A. Lécuyer, "wow! i have six fingers!: Would you accept structural changes of your hand in vr?," Frontiers, vol. 3, no. 27, p. 1, 2016.

[12] M. Botvinick, J. Cohen, et al., "Rubber hands' feel'touch that eyes see," Nature, vol. 391, no. 6669, pp. 756-756, 1998.

[13] A. Guterstam, V. I. Petkova, and H. H. Ehrsson, "The illusion of owning a third arm," PloS one, vol. 6, no. 2, p. e17208, 2011.

[14] B. van der Hoort, A. Guterstam, and H. H. Ehrsson, "Being barbie: the size of ones own body determines the perceived size of the world," PloS one, vol. 6, no. 5, p. e20195, 2011.

[15] R. Newport, R. Pearce, and C. Preston, "Fake hands in action: embodiment and control of supernumerary limbs," Experimental brain research, vol. 204, no. 3, pp. 385-395, 2010.
[16] A. Folegatti, A. Farne, R. Salemme, and F. De Vignemont, "The rubber hand illusion: Twosa company, but threesa crowd," Consciousness and cognition, vol. 21, no. 2, pp. 799-812, 2012.

[17] B. Vigaru, J. Sulzer, and R. Gassert, "Design and evaluation of a cable-driven fmri-compatible haptic interface to investigate precision grip control," IEEE Transactions on Haptics, vol. 9, pp. 20-32, Jan 2016.

[18] K. Chinzei, R. Kikinis, and F. A. Jolesz, MR Compatibility of Mechatronic Devices: Design Criteria, pp. 1020-1030. Berlin, Heidelberg: Springer Berlin Heidelberg, 1999.

[19] N. Vanello, V. Hartwig, M. Tesconi, E. Ricciardi, A. Tognetti, G. Zupone, R. Gassert, D. Chapuis, N. Sgambelluri, E. P. Scilingo, et al., "Sensing glove for brain studies: design and assessment of its compatibility for fmri with a robust test," IEEE/Asme Transactions on Mechatronics, vol. 13, no. 3, pp. 345-354, 2008.

[20] A. M. Dollar and R. D. Howe, "The highly adaptive sdm hand: Design and performance evaluation," The international journal of robotics research, vol. 29, no. 5, pp. 585-597, 2010.

[21] I. Hussain, G. Spagnoletti, G. Salvietti, and D. Prattichizzo, "an emg interface for the control of motion and compliance of a supernumerary robotic finger," Frontiers in Neurorobotics, vol. 10, 2016.

[22] Arduino, "Arduino uno, an open-source electronics prototyping platform." On-line: http://arduino.cc/.

[23] Immersion Technologies, "Cyberglove wireless system." on-line: http://www.cyberglovesystems.com/.

[24] R. W. Cox, "Afni: software for analysis and visualization of functional magnetic resonance neuroimages," Computers and Biomedical research, vol. 29, no. 3, pp. 162-173, 1996.

[25] M. Jenkinson, C. F. Beckmann, T. E. Behrens, M. W. Woolrich, and S. M. Smith, "Fsl," Neuroimage, vol. 62, no. 2, pp. 782-790, 2012.

[26] J. D. Power, K. A. Barnes, A. Z. Snyder, B. L. Schlaggar, and S. E. Petersen, "Spurious but systematic correlations in functional connectivity mri networks arise from subject motion," Neuroimage, vol. 59, no. 3, pp. 2142-2154, 2012.

[27] E. Abdi, E. Burdet, M. Bouri, and H. Bleuler, "Control of a supernumerary robotic hand by foot: An experimental study in virtual reality," PloS one, vol. 10, no. 7, p. e0134501, 2015.

[28] J. Tanji, "The supplementary motor area in the cerebral cortex," Neuroscience research, vol. 19, no. 3, pp. 251-268, 1994.

[29] S. H. Johnson-Frey, "The neural bases of complex tool use in humans," Trends in cognitive sciences, vol. 8, no. 2, pp. 71-78, 2004. 